\title{
Active Queue Management within Large-Scale Wired Networks
}

\author{
Ping-Min Hsu ${ }^{1}$, Chun-Liang Lin ${ }^{1}$, Ching-Han $\mathbf{Y u}^{2}$ \\ ${ }^{1}$ Department of Electrical Engineering, National Chung Hsing University, Taichung, Chinese Taipei \\ ${ }^{2}$ Department of Electrical Engineering, National Taiwan University, Chinese Taipei \\ E-mail: chunlin@dragon.nchu.edu.tw \\ Received March 3, 2011; revised March 24, 2011; accepted March 30, 2011
}

\begin{abstract}
Signal transmission control protocol sources with the objective of managing queue utilization and delay is actually a feedback control problem in active queue management (AQM) core routers. This paper extends AQM control design for single network systems to large-scale wired network systems with time delays at each communication channel. A system model consisted of several local networks is first constructed. The stability condition guaranteeing overall stability is subsequently derived using Lyapunov stability theory. The results developed have been successfully verified on a network simulator.
\end{abstract}

Keywords: Large-Scale System, Network Control, Stability, Time Delay

\section{Introduction}

Traffic characterization and modeling are generally recognized as two important steps toward analysis and control of network transmission performance. With the aid of the characterized model, control theory can be efficiently applied in solving the congestion control problem. There have been various delayed differential equation models developed in [1-3], in which the fluid-flow model utilized in [1] was previously proposed in [2]. Those characterized data traffic as fluid used a set of differential equations to describe the Active Queue Management (AQM) policy and the router queuing process. In [4], the end-to-end congestion control mechanisms were employed in transmission control protocol (TCP) flow control.

While a bunch of research focusing on modeling and analysis of network control systems have been published, most approaches addressed the issue for a single senderreceiver network connection [5].

This paper is motivated by the requirement of an approach for modeling and stabilization of large-scale communication networks. An extension of the fluidbased model in wireless networks was proposed in $[6,7]$, in which their parameters for representing the probability of data transmission failure was applied in system development. A system for mixed wired and wireless networks was concerned in [6], in which a more general fluid-based model compared with that in [1] was proposed. A robust $H_{\infty}$ controller design was proposed in [7], in which the stability analysis was conducted by Lyapunov theory and modern control theory was extended to deal with the network congestion problem. Furthermore, linear matrix inequalities were proposed in control design and less oscillation under TCP was achieved. Packet dropout not only affects stability in data transmission, but also plays an important role in the network control systems. The authors in [8-10] have investigated stability for the network control systems with packet dropout.

To the best of the authors' knowledge, while the Lyapunov stability theory has been widely applied to the stability analysis of large-scale systems [11-13], investigation for stability of the large-scale network control systems (LWNCSs) are quite rare. This motivates this research. The presented work shows that an appropriate control design based on an appropriate Lyapunov functional ensuring asymptotic stability of the LWNCS can be constructed when there are a large number of signal flows.

The major contributions can be summarized as follows.

1) A control strategy for congestion control in the large-scale wired network is constructed.

2) All local networks are interconnected according to the dropping probability. That is, how the local net- 
works mutually affect each other within a wired network scheme is modeled via a particular form of the dropping probability.

An example is given to demonstrate validity of the result using the network simulation platform NS2 [14].

\section{Modeling of Large-Scale Wired Network}

The large-scale wired network under consideration is as illustrated in Figure 1, which consists of $s$ locally wired networks within TCP under random early detection (RED). In each TCP loop, signal packets are transmitted from a sender to a receiver, which is also the sender to next TCP loop. Furthermore, they are sequentially passed from $T C P_{1}$ to $T C P_{s}$. Some packets are dropped in the congested router under RED, in which the dropping probability is computed by the proposed controller. Regarding the topology illustrated in Figure 1, the current dropping probability not only determines the seriousness of transmission congestion in the present router, but also affects those in other congested routers in the large-scale wired network.

Referring to [1], a simplified version of a fluid-flow model of TCP behavior involving the key network variables could be modeled as

$$
\begin{gathered}
\dot{W}(t)=\frac{1}{R(t)}-\frac{W(t)}{2} \frac{W(t-R(t))}{R(t-R(t))} p(t-R(t)), \\
W(0)=W_{0} \\
\dot{q}(t)= \begin{cases}N(t) W(t) / R(t)-C, \quad q(0)=q_{0}, & q>0 \\
\max (0, N(t) W(t) / R(t)-C), & q=0\end{cases}
\end{gathered}
$$

where $W$ is the average TCP window size (packets), $q$ is the average queue length (packets/sec), $R(t)$ is the round trip time (RTT), $C$ is the link capacity (packets/sec), $N$ is the loading factor (the number of TCP sessions), and $p \in[0,1]$ is the probability of packet mark. The queue length $q \in\left[0, q_{m}\right]$ and the window size $W \in\left[0, W_{m}\right]$ where $q_{m}$ and $W_{m}$ denote the buffer capacity and the maximum window size respectively. Let $R(t)$ denote the RTT for $t \geq 0$ and is assumed to be

$$
R(t)=q(t) / C+T_{p}
$$

where $T_{p}$ is the fixed propagation delay and $q(t) / C$ models the queuing delay. One is referred to [1] for the details of the mathematical modeling of the fluid-flow model of TCP.

Suppose that each dynamic model of TCP constructs a sub-network, which belongs to an interconnected bottleneck network, then a large-scale wired network composed of $s$ interconnected bottleneck networks $S_{i}$, $i=1,2, \cdots, s$ can be shown as in Figure 2, where $R_{i}(t)=\bar{q}_{i}(t) / C_{i}+T_{i p}, i=1, \cdots, s$, with $T_{i p}$ being the propagation delay for $T C P_{i}$ and $\bar{q}_{i}(t)=q_{1 i}(t)+q_{2 i}(t)$ $+\cdots+q_{s i}(t)$. From (1) and (2), the large-scale linearized differential equations can be written in the matrix form as

$$
\begin{aligned}
& {\left[\begin{array}{l}
\delta \dot{w}_{i}(t) \\
\delta \dot{q}_{i}(t)
\end{array}\right]} \\
& =\left[\begin{array}{cc}
\frac{-N_{i}}{R_{i 0}^{2} C_{i}} & \frac{-1}{R_{i 0}^{2} C_{i}} \\
\frac{N_{i}}{R_{i 0}} & \frac{-1}{R_{i 0}}
\end{array}\right]\left[\begin{array}{l}
\delta w_{i}(t) \\
\delta q_{i}(t)
\end{array}\right]+\left[\begin{array}{c}
\frac{-R_{i 0}^{2} C_{i}^{2}}{2 N_{i}^{2}} \\
0
\end{array}\right] \delta p_{i}\left(t, R_{i 0}\right) \\
& +\left[\begin{array}{cc}
\frac{-N_{i}}{R_{i 0}^{2} C_{i}} & \frac{1}{R_{i 0}^{2} C_{i}} \\
0 & 0
\end{array}\right]\left[\begin{array}{l}
\delta w_{i}\left(t-R_{i 0}\right) \\
\delta q_{i}\left(t-R_{i 0}\right)
\end{array}\right], i=1,2, \cdots, s
\end{aligned}
$$

with each sub-network being interconnected through

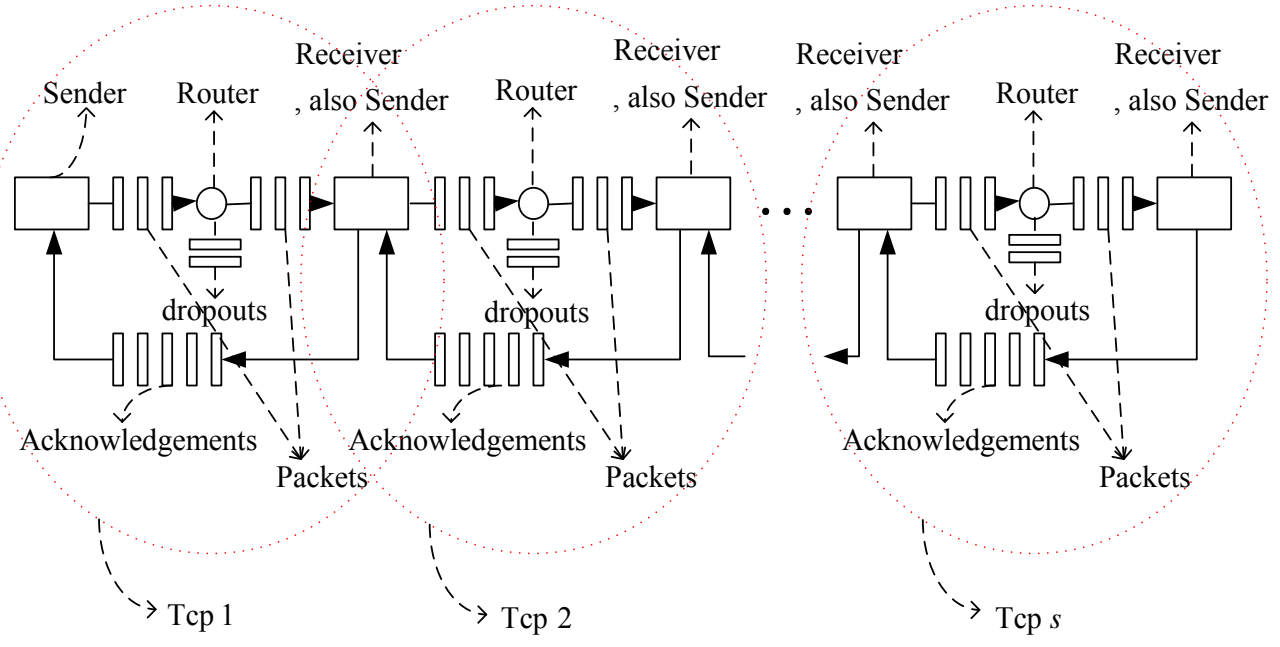

Figure 1. Topology of the large-scale wired network under consideration. 


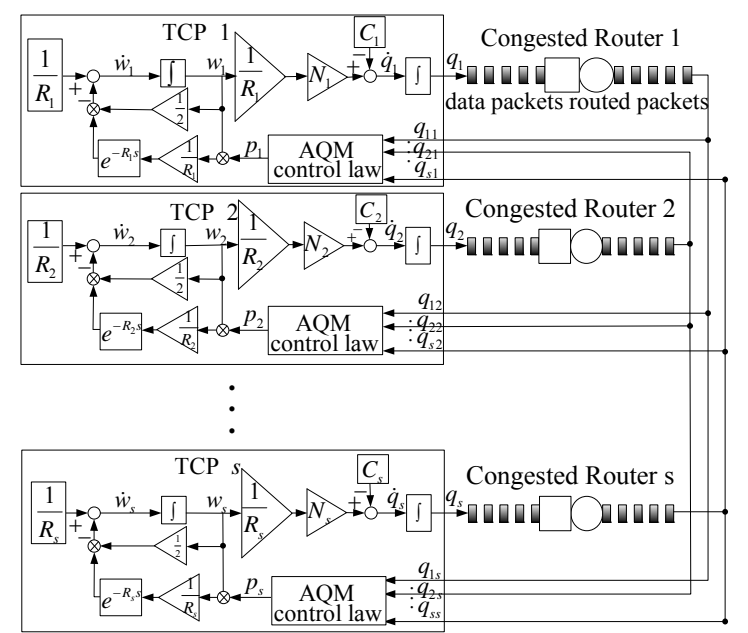

Figure 2. Block diagram of the large-scale wired network.

$$
\begin{aligned}
& \delta p_{i}\left(t, R_{i o}\right)=K_{p i} \sum_{j=1}^{s} \mathrm{~d}_{i j}\left[q_{0}+\delta q_{j}\left(t-h_{i j}\right)\right] \\
& -p_{i 0}-\int_{-R_{i 0}}^{0} \frac{\partial}{\partial v} \delta p_{i}(t+v) \mathrm{d} v+K_{p i} \sum_{j=1}^{s} d_{i j} \overline{\mathbf{u}}_{i}(t) \\
& -\left[2 N_{i}^{3} \int_{-R_{i 0}}^{0} \frac{\partial}{\partial v} \delta w_{i}(t+v) \mathrm{d} v / R_{i 0}^{3} C_{i}^{3}\right. \\
& \left.-2 N_{i}^{2} \int_{-R_{i 0}}^{0} \frac{\partial}{\partial v} \delta q_{i}(t+v) \mathrm{d} v / R_{i 0}^{3} C_{i}^{3}\right], i, j=1,2 \cdots, s
\end{aligned}
$$

where $h_{i j}$ is the transmission time between the bottleneck networks $S_{i}$ and $S_{j} ; \delta w_{i}=w_{i}-w_{i 0}, \delta q_{i}=q_{i}-q_{0}$, $\delta q_{j}=q_{j}-q_{0}$, and $\delta p_{i}=p_{i}-p_{i 0} ;\left(w_{i 0}, q_{0}, p_{i 0}\right)$ denoting our operating point; $q_{i}$ and $q_{j}$ denote data packets belonging to the $i$ th and $j$ th sub-networks respectively; $w_{i}$ denotes the $i$ th expected TCP sending window; $C_{i}$ denotes the link capacity; $N_{i}$ denotes the number of the ith TCP sessions; $R_{i 0}=q_{0} / C_{i}+T_{i p} ; p_{i}$ is the probability of packet mark; RED consists of a proportional controller and packet-marking profile, shown as in Figure 3, where $q^{\min }, q^{\max }$ and $p^{\max }$ are configurable parameters and $K_{p i}$ denotes the slope of the active queue management control law which computes $p_{i}$ as the function of the measured queue length $\bar{q}_{i}$ by the AQM policy; $d_{i j}$ is the flow distribution ratio from the bottleneck network $S_{j}$ to $S_{i}$. If $\bar{q}_{i}(t)$ is bounded by $q^{\text {min }}$ and $q^{\max }$, the packet-marking probability is given by

$$
\begin{aligned}
p_{i}= & K_{p i} \sum_{j=1}^{s} d_{i j}\left[q_{0}+\delta q_{j}\left(t-h_{i j}\right)\right] \\
& -\int_{-R_{i 0}}^{0} \frac{\partial}{\partial v} \delta p_{i}(t+v) \mathrm{d} v+K_{p i} \sum_{j=1}^{s} \mathrm{~d}_{i j} \overline{\boldsymbol{u}}_{i}(t) \\
& -\left[\frac{2 N_{i}^{3}}{R_{i 0}^{3} C_{i}^{3}} \int_{-R_{i 0}}^{0} \frac{\partial}{\partial v} \delta w_{i}(t+v) \mathrm{d} v\right. \\
& \left.-\frac{2 N_{i}^{2}}{R_{i 0}^{3} C_{i}^{3}} \cdot \int_{-R_{i 0}}^{0} \frac{\partial}{\partial v} \delta q_{i}(t+v) \mathrm{d} v\right]
\end{aligned}
$$

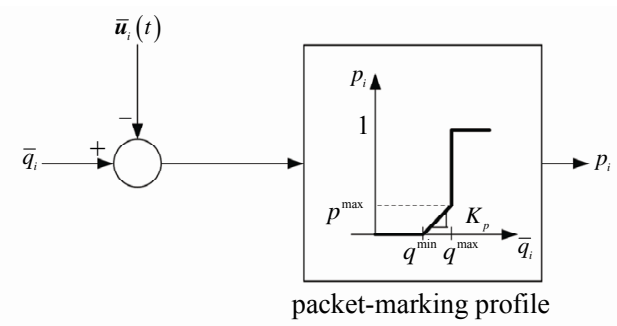

Figure 3. RED drop function.

The form of (5) is determined based on the following considerations.

1) While each sub-network is interconnected through (5), the feedback signal applied for the controller is treated as the combination of all the queue lengths in the large-scale network. Thus, the term $\sum_{j=1}^{s} d_{i j}\left[q_{0}+\delta q_{j}\left(t-h_{i j}\right)\right]$ in (5) was claimed. It was further multiplied with $K_{p i}$, which denotes the slope of the active queue management control law.

2) The term $\int_{-R_{i 0}}^{0} \frac{\partial}{\partial v} \delta p_{i}(t+v) \mathrm{d} v$ denotes the difference between the current and delayed values of the dropping probability adopted in the ith TCP. Considering the fluid-flow model, the current queue length variation is affected by the delayed dropping probability while the current one is applied in real world. Considering this fact, while constructing (5), the term $-\int_{-R_{i 0}}^{0} \frac{\partial}{\partial v} \delta p_{i}(t+v) \mathrm{d} v$ is added.

3) $p_{i 0}$ is added to cancel $p_{i 0}$ while computing $p_{i}$ where $p_{i 0}=p_{i}-\delta p_{i}$.

4) Additional terms in the AQM controller law are added to obtain the transformed system (6) with the controller (7).

5) Now, substituting $\delta p_{i}\left(t, R_{i 0}\right)$, defined by (5), into (4) gives a new system. Based on (4), a large-scale wired network system consisting of $s$ local networks can be expressed in the state-space representation as follows

$$
\dot{x}_{i}(t)=\boldsymbol{A}_{i i} \boldsymbol{x}_{i}(t)+\boldsymbol{B}_{i} \boldsymbol{u}_{i}(t), \quad i=1,2, \cdots, s
$$

where $\boldsymbol{x}_{i}(t)=\left[\begin{array}{ll}\delta w_{i}(t) & \delta q_{i}(t)\end{array}\right]^{T}$,

$$
\begin{aligned}
& \boldsymbol{A}_{i i}=\left[\begin{array}{cc}
\frac{-2 N_{i}}{R_{i 0}^{2} C_{i}} & 0 \\
\frac{N_{i}}{R_{i 0}} & \frac{-1}{R_{i 0}}
\end{array}\right], \boldsymbol{B}_{i}=\left[\begin{array}{c}
\frac{-R_{i 0} C_{i}^{2}}{2 N_{i}^{2}} \\
0
\end{array}\right], \text { and } \\
& \boldsymbol{u}_{i}(t)=\sum_{j=1}^{s} F_{i j} x_{j}\left(t-h_{i j}\right)+\Psi_{i}\left(\overline{\boldsymbol{u}}_{i}(t)\right), i, j=1,2, \cdots, s
\end{aligned}
$$

with $\quad \boldsymbol{F}_{i j}=\left[\begin{array}{ll}0 & K_{p i} d_{i j}\end{array}\right]$ and $\overline{\boldsymbol{u}}_{i}(t)=-K_{i} \boldsymbol{x}_{i}(t)$ being the AQM control law for the ith subsystem, and 


$$
\begin{aligned}
\Psi_{i}\left(\overline{\boldsymbol{u}}_{i}(t)\right)= & K_{p i} \sum_{j=1}^{s} d_{i j}\left(q_{0}+\overline{\boldsymbol{u}}_{i}(t)\right)-p_{i 0} \\
& -\int_{-R_{i 0}}^{0} \frac{\partial}{\partial v} \delta p_{i}(t+v) \mathrm{d} v \\
& -\left[\frac{2 N_{i}^{3}}{R_{i 0}^{3} C_{i}^{3}} \int_{-R_{i 0}}^{0} \frac{\partial}{\partial v} \delta w_{i}(t+v) \mathrm{d} v\right. \\
& \left.-\frac{2 N_{i}^{2}}{R_{i 0}^{3} C_{i}^{3}} \cdot \int_{-R_{i 0}}^{0} \frac{\partial}{\partial v} \delta q_{i}(t+v) \mathrm{d} v\right]
\end{aligned}
$$

Equation (6) represents the linearized system of the large-scale wired network while considering coupling effects induced by the locally wired networks. The term $\boldsymbol{u}_{i}(t)$ implies the dropping probability while concerning the interconnection of local networks. The term $\overline{\boldsymbol{u}}_{i}(t)$ is to be determined in the stability analysis. Equation (7) is treated as the control input such that $p_{i}(t)=\boldsymbol{u}_{i}(t)+p_{i 0}$ is applied as the dropping probability.

$\boldsymbol{u}_{i}(t)$ can be derived in the following steps:

Step 1) Consider (5), which models how local networks may mutually affect each other in the large-scale wired networking environment.

Step 2)Substitute $\delta p_{i}\left(t, R_{i 0}\right)$ into (4) and use $\boldsymbol{x}_{i} \cong$ $x_{i}\left(t-R_{i 0}\right)$ to obtain system of (6) with the controller (7).

From (6) it is easily seen that $\left(\boldsymbol{A}_{i i}, \boldsymbol{B}_{i}\right)$ is controllable. The action of the AQM control law is to mark packets as a function of the measured queue length $q$. The plant dynamics given by (6) relates reveals the packet-marking probability dynamically affects the queue length.

Now, let the set of feasible operating conditions $q_{0} \in\left[0, q_{m}\right], \quad w_{i 0} \in\left[0, W_{m}\right]$, and $p_{i 0} \in[0,1]$. Assume that $\delta w_{i}, \delta q_{i}$ and $\delta p_{i}$ are continuously differentiable on $\left[-R_{i 0}, 0\right]$, i.e. $\left|\frac{\partial \delta w_{i}}{\partial v}\right| \leq b_{W}<\infty,\left|\frac{\partial \delta q_{i}}{\partial v}\right| \leq b_{q}<\infty$, $\left|\frac{\partial \delta p_{i}}{\partial v}\right| \leq b_{p}<\infty, \quad \forall v \in\left[-R_{i 0}, 0\right]$.

Therefore

$$
\begin{aligned}
& \left|\int_{-R_{i 0}}^{0} \frac{\partial}{\partial v} \delta w_{i}(t+v) \mathrm{d} v\right| \leq b_{w} R_{i 0}, \\
& \left|\int_{-R_{i 0}}^{0} \frac{\partial}{\partial v} \delta q_{i}(t+v) \mathrm{d} v\right| \leq b_{q} R_{i 0}, \\
& \left|\int_{-R_{i 0}}^{0} \frac{\partial}{\partial v} \delta p_{i}(t+v) \mathrm{d} v\right| \leq b_{p} R_{i 0} .
\end{aligned}
$$

Furthermore

$$
\begin{aligned}
\overline{\boldsymbol{u}}_{i}(t) \Psi_{i}\left(\overline{\boldsymbol{u}}_{i}(t)\right)= & K_{p i} \sum_{j=1}^{s} d_{i j} \overline{\boldsymbol{u}}_{i}^{2}(t)+K_{p i} q_{0} \sum_{j=1}^{s} d_{i j} \overline{\mathbf{u}}_{i}(t) \\
& -\left(p_{i 0}+g_{i}(t)\right) \overline{\boldsymbol{u}}_{i}(t)
\end{aligned}
$$

where

$$
\begin{aligned}
g_{i}(t) & =\frac{2 N_{i}^{3}}{R_{i 0}^{3} C_{i}^{3}} \int_{-R_{i 0}}^{0} \frac{\partial}{\partial v} \delta w_{i}(t+v) \mathrm{d} v \\
& -\frac{2 N_{i}^{2}}{R_{i 0}^{3} C_{i}^{3}} \int_{-R_{i 0}}^{0} \frac{\partial}{\partial v} \cdot \delta q_{i}(t+v) \mathrm{d} v \\
& +\int_{-R_{i 0}}^{0} \frac{\partial}{\partial v} \delta p_{i}(t+v) \mathrm{d} v
\end{aligned}
$$

It is seen that $-g_{i m} \leq g_{i}(t) \leq g_{i m}$ with $g_{i m}=\frac{2 N_{i}^{3}}{R_{i 0}^{2} C_{i}^{3}} b_{W}$ $+\frac{2 N_{i}^{2}}{R_{i 0}^{2} C_{i}^{3}} b_{q}+R_{i 0} b_{p}$. The gain $K_{p i}$ is chosen as

$$
K_{p i}=\left\{\begin{array}{l}
\frac{1}{q_{0} \sum_{j=1}^{s} d_{i j}}\left(p_{i 0}+g_{i m}\right), \text { when } \overline{\boldsymbol{u}}_{i} \geq 0, \\
\frac{1}{q_{0} \sum_{j=1}^{s} d_{i j}}\left(p_{i 0}-g_{i m}\right), \text { otherwise, }
\end{array} \quad i=1,2, \cdots, s(9)\right.
$$

This ensures $\overline{\boldsymbol{u}}_{i}(t) \Psi_{i}\left(\overline{\boldsymbol{u}}_{i}(t)\right)>0, \forall \overline{\boldsymbol{u}}_{i}(t)$.

With regard to the above system one can ensure that $\Psi_{i}(\cdot), \quad i=1,2, \cdots, s$ satisfy

$$
\gamma_{i} \overline{\boldsymbol{u}}_{i}^{2}(t) \leq \overline{\boldsymbol{u}}_{i}(t) \Psi_{i}\left(\overline{\boldsymbol{u}}_{i}(t)\right), \forall \overline{\boldsymbol{u}}_{i}(t) \in R, i=1,2, \cdots, s
$$

From (8) and (9), the gain reduction tolerance is given by $\gamma_{i}=K_{p i} \sum_{j=1}^{s} d_{i j}$ where $d_{i j}, j=1, \cdots, s$ are chosen such that $\gamma_{i}=K_{p i} \sum_{j=1}^{s} d_{i j}<1$.

\section{Stability of LWNCS}

The following theorem states the main result which characterizes the stability condition for the LWNCS.

Lemma: For any scalar $\varepsilon>0$ and any real vectors $\boldsymbol{X}$ and $\boldsymbol{Y}$ with appropriate dimension, then

$$
\boldsymbol{X}^{\mathrm{T}} \boldsymbol{Y}+\boldsymbol{Y}^{\mathrm{T}} \boldsymbol{X} \leq \varepsilon \boldsymbol{X}^{\mathrm{T}} \boldsymbol{X}+\frac{1}{\varepsilon} \boldsymbol{Y}^{\mathrm{T}} \boldsymbol{Y}
$$

Theorem: The large-scale wired network system described by (6), which satisfies (10), would be asymptotically stable, if the state feedback control law of each sub-network is given by

$$
\overline{\boldsymbol{u}}_{i}(t)=-\frac{1}{2} \rho_{i} \boldsymbol{B}_{i}^{T} \boldsymbol{P}_{i} \boldsymbol{x}_{i}(t), \quad i=1,2, \cdots, s
$$

where $\rho_{i}=1+\psi_{i} / \gamma_{i}$ with $\psi_{i}$ satisfying

$$
2-\gamma_{i}-2 \sqrt{1-\gamma_{i}}<\psi_{i}<2-\gamma_{i}+2 \sqrt{1-\gamma_{i}}
$$

and $\boldsymbol{P}_{i}=\boldsymbol{P}_{i}^{T}>0$ satisfies the following Riccati matrix inequality:

$$
\begin{aligned}
& \boldsymbol{A}_{i i}^{\mathrm{T}} \boldsymbol{P}_{i}+\boldsymbol{P}_{i} \boldsymbol{A}_{i i}+\boldsymbol{P}_{i}\left[s \varepsilon_{1} \boldsymbol{I}_{n}-\left(\gamma_{i}+\phi_{i}\right) \boldsymbol{B}_{i} \boldsymbol{B}_{i}^{\mathrm{T}}\right] \boldsymbol{P}_{i} \\
& +\left(s \alpha_{i}+1\right) \boldsymbol{I}_{n}<0
\end{aligned}
$$


in which $\varepsilon_{1}$ is a positive constant, $\phi_{i}=\psi_{i}-\left(\gamma_{i}+\psi_{i}\right)^{2} / 4$ and $\alpha_{i}:=\max \left\{\alpha_{j i}, j=1,2, \cdots, s\right\}$ with $\alpha_{j i}$ satisfying

$$
\begin{aligned}
& \alpha_{i j} \boldsymbol{I}_{n}>\frac{1}{\varepsilon_{1}} \boldsymbol{F}_{i j}^{T} \boldsymbol{B}_{i}^{T} \boldsymbol{B}_{i} \boldsymbol{F}_{i j}, \quad \forall i, j \\
& \boldsymbol{V}_{i}\left(\boldsymbol{x}_{i}\right)=\sum_{i=1}^{s}\left[\boldsymbol{x}_{i}^{\mathrm{T}}(t) \boldsymbol{P}_{i} \boldsymbol{x}_{i}(t)+\sum_{j=1}^{s} \alpha_{i j} \int_{t-h_{i j}}^{t} \boldsymbol{x}_{j}^{\mathrm{T}}(\tau) \boldsymbol{x}_{j}(\tau) \mathrm{d} \tau+\int_{0}^{t} \boldsymbol{x}_{i}^{T}(\tau) \boldsymbol{x}_{i}(\tau) \mathrm{d} \tau+\gamma_{i}^{2} \int_{0}^{t} \overline{\boldsymbol{u}}_{i}^{2}(\tau) \mathrm{d} \tau\right]
\end{aligned}
$$

Proof: The stability analysis is derived around the origin $\delta q_{i}=\delta w_{i}=0$ of (4), i.e. the equilibrium point $\left(q_{0}, p_{i 0}, w_{i 0}\right)$ of the fluid-flow model. Regarding the problem, we define an appropriate Lyapunov functional

for $i \in\{1,2, \cdots, s\}$. The set $\left(q_{0}, p_{i 0}, w_{i 0}\right)$ chosen as the equilibrium point of the fluid-flow model corresponding

to $T C P_{i}$ is determined by

$$
\frac{1}{R_{i 0}}-\frac{w_{i 0}^{2}}{2 R_{i 0}} p_{i 0}=0, \frac{N_{i}}{R_{i 0}} w_{i 0}-C_{i}=0, \quad R_{i 0}=T_{i p}+\frac{q_{0}}{C_{i}},\left|p_{i 0}\right| \leq 1
$$

It is obtained that $\boldsymbol{V}_{i}(0)=0$ while $\overline{\boldsymbol{u}}_{i}=0$ and $\boldsymbol{V}_{i}\left(x_{i}\right) \neq 0$ for $\boldsymbol{x}_{i} \neq 0$. Taking differentiation with re-

spect to time $t$ and using (6) while ignoring $-\sum_{i=1}^{s} \boldsymbol{x}_{i}^{\mathrm{T}}(0) \boldsymbol{x}_{i}(0)$ and $-\sum_{i=1}^{s} \overline{\boldsymbol{u}}_{i}^{2}(0)$ gives

$$
\begin{aligned}
\dot{V}_{i}\left(\boldsymbol{x}_{i}\right) \leq & \sum_{i=1}^{s} \boldsymbol{x}_{i}^{\mathrm{T}}(t)\left[\boldsymbol{A}_{i i}^{\mathrm{T}} \boldsymbol{P}_{i}+\boldsymbol{P}_{i} \boldsymbol{A}_{i i}+I_{n}\right] \boldsymbol{x}_{i}(t) \\
& +\sum_{i=1}^{s} \gamma_{i}^{2} \overline{\boldsymbol{u}}_{i}^{2}(t)+2 \sum_{i=1}^{s} \boldsymbol{x}_{i}^{\mathrm{T}}(t) \boldsymbol{P}_{i} \boldsymbol{B}_{i} \boldsymbol{F}_{i j} \boldsymbol{x}_{j}\left(t-h_{i j}\right) \\
& +2 \sum_{i=1}^{s} \boldsymbol{x}_{i}^{\mathrm{T}}(t) \boldsymbol{P}_{i} \boldsymbol{B}_{i} \Psi_{i}\left(\overline{\boldsymbol{u}}_{i}(t)\right) \\
& +\sum_{i=1}^{s} \sum_{j=1}^{s} \alpha_{i j}\left[\boldsymbol{x}_{j}^{\mathrm{T}}(t) \boldsymbol{x}_{j}(t)-\boldsymbol{x}_{j}^{\mathrm{T}}\left(t-h_{i j}\right) \boldsymbol{x}_{j}\left(t-h_{i j}\right)\right]
\end{aligned}
$$

It is easily obtained from (11) that

$$
\begin{aligned}
& \sum_{i=1}^{s} \sum_{j=1}^{s}\left\{\boldsymbol{x}_{j}^{\mathrm{T}}\left(t-h_{i j}\right) \boldsymbol{F}_{i j}^{\mathrm{T}} \boldsymbol{B}_{i}^{\mathrm{T}} \boldsymbol{P}_{i} \boldsymbol{x}_{i}(t)+\boldsymbol{x}_{i}^{\mathrm{T}}(t) \boldsymbol{P}_{i} \boldsymbol{B}_{i} \boldsymbol{F}_{i j} \boldsymbol{x}_{j}\left(t-h_{i j}\right)\right\} \\
& \leq \sum_{i=1}^{s} \sum_{j=1}^{s}\left[\frac{1}{\varepsilon_{1}} \boldsymbol{x}_{j}^{\mathrm{T}}\left(t-h_{i j}\right) \boldsymbol{F}_{i j}^{\mathrm{T}} \boldsymbol{B}_{i}^{\mathrm{T}} \boldsymbol{B}_{i} \boldsymbol{F}_{i j} \boldsymbol{x}_{j}\left(t-h_{i j}\right)\right. \\
& \left.+\varepsilon_{1} \boldsymbol{x}_{i}^{\mathrm{T}}(t) \boldsymbol{P}_{i} \boldsymbol{P}_{i} \boldsymbol{x}_{i}(t)\right] \\
& =\sum_{i=1}^{s} \sum_{j=1}^{s}\left[\frac{1}{\varepsilon_{1}} \boldsymbol{x}_{j}^{\mathrm{T}}\left(t-h_{i j}\right) \boldsymbol{F}_{i j}^{\mathrm{T}} \boldsymbol{B}_{i}^{\mathrm{T}} \boldsymbol{B}_{i} \boldsymbol{F}_{i j} \boldsymbol{x}_{j}\left(t-h_{i j}\right)\right] \\
& +s \varepsilon_{1} \sum_{i=1}^{s} \boldsymbol{x}_{i}^{\mathrm{T}}(t) \boldsymbol{P}_{i} \boldsymbol{P}_{i} \boldsymbol{x}_{i}(t)
\end{aligned}
$$

$$
\begin{aligned}
& 2 \sum_{i=1}^{s} \boldsymbol{x}_{i}^{\mathrm{T}}(t) \boldsymbol{P}_{i} \boldsymbol{B}_{i} \Psi_{i}\left(\overline{\boldsymbol{u}}_{i}(t)\right) \\
& \leq-\gamma_{i} \rho_{i} \sum_{i=1}^{s} \boldsymbol{x}_{i}^{\mathrm{T}}(t) \boldsymbol{P}_{i} \boldsymbol{B}_{i} \boldsymbol{B}_{i}^{\mathrm{T}} P_{i} \boldsymbol{x}_{i}(t)
\end{aligned}
$$

Furthermore

$$
\sum_{i=1}^{s} \sum_{j=1}^{s} \alpha_{i j} \boldsymbol{x}_{j}^{\mathrm{T}}(t) \boldsymbol{x}_{j}(t) \leq s \sum_{i=1}^{s} \alpha_{i} \boldsymbol{x}_{i}^{\mathrm{T}}(t) \boldsymbol{x}_{i}(t)
$$

Therefore, it can be obtained that

$$
\begin{aligned}
\dot{\boldsymbol{V}}_{i}\left(\boldsymbol{x}_{i}\right) & \leq \sum_{i=1}^{s} \boldsymbol{x}_{i}^{\mathrm{T}}(t)\left[\boldsymbol{A}_{i i}^{\mathrm{T}} \boldsymbol{P}_{i}+\boldsymbol{P}_{i} \boldsymbol{A}_{i i}+\left(s \alpha_{i}+1\right) \boldsymbol{I}_{n}+s \varepsilon_{1} \boldsymbol{P}_{i}^{2}\right. \\
& \left.+\left(\frac{1}{4}\left(\gamma_{i}+\psi_{i}\right)^{2}-\psi_{i}-\gamma_{i}\right) \boldsymbol{P}_{i} \boldsymbol{B}_{i} \boldsymbol{B}_{i}^{\mathrm{T}} \boldsymbol{P}_{i}\right] \boldsymbol{x}_{i}(t) \\
& +\sum_{i=1}^{s} \sum_{j=1}^{s} \boldsymbol{x}_{j}^{\mathrm{T}}\left(t-h_{i j}\right)\left(\frac{1}{\varepsilon_{1}} \boldsymbol{F}_{i j}^{\mathrm{T}} \boldsymbol{B}_{i}^{\mathrm{T}} \boldsymbol{B}_{i} \boldsymbol{F}_{i j}-\alpha_{i j} \boldsymbol{I}_{n}\right) \boldsymbol{x}_{j}\left(t-h_{i j}\right)
\end{aligned}
$$

$$
\gamma_{i} \rho_{i} \boldsymbol{x}_{i}^{\mathrm{T}}(t) \boldsymbol{P}_{i} \boldsymbol{B}_{i} \boldsymbol{B}_{i}^{\mathrm{T}} \boldsymbol{P}_{i} x_{i}(t) / 2 \leq-\boldsymbol{x}_{i}^{\mathrm{T}}(t) \boldsymbol{P}_{i} \boldsymbol{B}_{i} \Psi_{i}\left(\overline{\boldsymbol{u}}_{i}(t)\right)
$$

i.e.

From (12)-(15), it can be concluded that

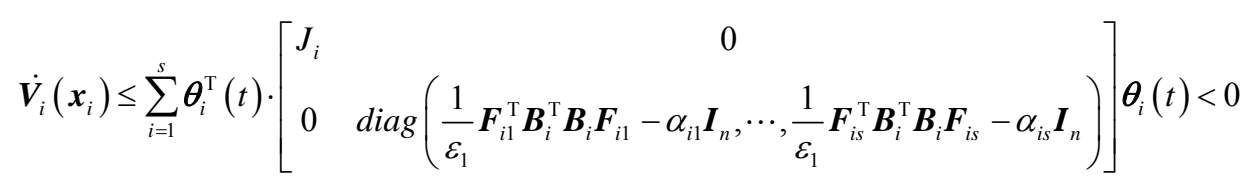


where $\boldsymbol{\theta}_{i}(t)=\left[\begin{array}{llll}\boldsymbol{x}_{i}^{\mathrm{T}}(t) & \boldsymbol{x}_{1}^{\mathrm{T}}\left(t-h_{i 1}\right) & \cdots & \boldsymbol{x}_{s}^{\mathrm{T}}\left(t-h_{i s}\right)\end{array}\right]^{\mathrm{T}}$,

$$
\boldsymbol{J}_{i}=\boldsymbol{A}_{i i}^{\mathrm{T}} \boldsymbol{P}_{i}+\boldsymbol{P}_{i} \boldsymbol{A}_{i i}+\boldsymbol{P}_{i}\left[s \varepsilon_{1} \boldsymbol{I}_{n}-\left(\gamma_{i}+\phi_{i}\right) \boldsymbol{B}_{i} \boldsymbol{B}_{i}^{\mathrm{T}}\right] \boldsymbol{P}_{i}+\left(s \alpha_{i}+1\right) \boldsymbol{I}_{n}<0
$$

and $\quad \alpha_{i j} \boldsymbol{I}_{n}>\boldsymbol{F}_{i j}^{\mathrm{T}} \boldsymbol{B}_{i}^{\mathrm{T}} \boldsymbol{B}_{i} \boldsymbol{F}_{i j} / \varepsilon_{1}, \forall i, j \quad$ and $\quad \psi_{i}$ satisfies (13). This completes the proof.

\section{Design Process}

Control design procedure is given bellows to summarize the previous analysis.

Step 1) Set parameters of the LWNS including $C_{i}$, $N_{i}, R_{i 0}, T_{i p}, b_{w}, b_{q}$, and $b_{p}$. The system model of (6) is then constructed.

Step 2) Consider the model defined by (6) and (7) with the positive constant $\varepsilon_{1}$, the parameter $\alpha_{i j}$ is chosen to satisfy (15).

Step 3) Find $a_{i}, g_{i m}, \gamma_{i}, \psi_{i}, \rho_{i}$ and to solve for the Riccati matrix inequality (14). The solution $\boldsymbol{P}_{i}=\boldsymbol{P}_{i}^{\mathrm{T}}>0$ can be calculated by transforming (14) into linear matrix inequalities and solved via the available computational software.

Step 4) Obtain the control gain $K_{i}=\rho_{i} \boldsymbol{B}_{i}^{\mathrm{T}} \boldsymbol{P}_{i} / 2$. The AQM control law $\boldsymbol{u}_{i}(t)$ is determined by (7) with $\overline{\boldsymbol{u}}_{i}(t)=-\boldsymbol{K}_{i} \boldsymbol{x}_{i}(t)$.

\section{Illustrative Example}

Consider a large-scale wired network described by (4) with $q_{0}=175$ packets, $N_{1}=60, N_{2}=50, N_{3}=20$, $N_{4}=5, \quad R_{10}=0.2 \mathrm{~s}, \quad R_{20}=0.1 \mathrm{~s}, \quad R_{30}=0.1 \mathrm{~s}$, $R_{40}=0.1 \mathrm{~s}, \quad C_{1}=3,750$ packets $/ \mathrm{s}, C_{2}=4,000$ packets $/ \mathrm{s}, \quad C_{3}=4,000$ packets $/ \mathrm{s}, \quad C_{4}=4,000$ packets $/ \mathrm{s}$, and $\varepsilon_{1}=0.001$. The large-scale wired network consists of four sub-networks described, respectively, by

$$
\begin{aligned}
& \dot{\boldsymbol{x}}_{1}(t)=\left[\begin{array}{cc}
-8 & 0 \\
300 & -5
\end{array}\right] \boldsymbol{x}_{1}(t)+\left[\begin{array}{c}
-390 \\
0
\end{array}\right]\left[\sum_{j=1}^{4} A_{1 j} \boldsymbol{x}_{j}\left(t-h_{1 j}\right)+\Psi_{1}\left(\overline{\boldsymbol{u}}_{1}(t)\right)\right], \\
& \dot{\boldsymbol{x}}_{2}(t)=\left[\begin{array}{cc}
-2.5 & 0 \\
500 & -100
\end{array}\right] \boldsymbol{x}_{2}(t)+\left[\begin{array}{c}
-320 \\
0
\end{array}\right]\left[\sum_{j=1}^{4} A_{2 j} \boldsymbol{x}_{j}\left(t-h_{2 j}\right)+\Psi_{2}\left(\overline{\boldsymbol{u}}_{2}(t)\right)\right], \\
& \dot{\boldsymbol{x}}_{3}(t)=\left[\begin{array}{cc}
-1 & 0 \\
200 & -100
\end{array}\right] \boldsymbol{x}_{3}(t)+\left[\begin{array}{c}
-889 \\
0
\end{array}\right]\left[\sum_{j=1}^{4} A_{3 j} \boldsymbol{x}_{j}\left(t-h_{3 j}\right)+\Psi_{3}\left(\overline{\boldsymbol{u}}_{3}(t)\right)\right], \\
& \dot{\boldsymbol{x}}_{4}(t)=\left[\begin{array}{cc}
-0.25 & 0 \\
50 & -10
\end{array}\right] \boldsymbol{x}_{4}(t)+\left[\begin{array}{c}
-32000 \\
0
\end{array}\right]\left[\sum_{j=1}^{4} A_{4 j} \boldsymbol{x}_{j}\left(t-h_{4 j}\right)+\Psi_{4}\left(\overline{\boldsymbol{u}}_{4}(t)\right)\right]
\end{aligned}
$$

Select $\alpha_{i j}=0.04$ for each $i \in\{1,2,3,4\}$ and assume $b_{W}=b_{p}=b_{q}=200$ so that $g_{1 m}=40, g_{2 m}=g_{3 m}$ $=g_{4 m}=20$. Referring to [1], $w_{i 0}=R_{i 0} C_{i} / N_{i}$ and $w_{i 0}^{2} p_{i 0}=2$. From $\gamma_{i}=p_{i 0}+g_{i m} / q_{0}$ then $\gamma_{1}=0.2$, $\gamma_{2}=\gamma_{3}=\gamma_{4}=0.1$, and $\psi_{1}=\psi_{2}=\psi_{3}=\psi_{4}=0.4$ are chosen to meet (13). Solving for the Riccati matrix inequality (14) gives

$$
\begin{aligned}
& \boldsymbol{P}_{1}=\left[\begin{array}{ll}
0.321 & 0.017 \\
0.017 & 0.002
\end{array}\right], \boldsymbol{P}_{2}=\left[\begin{array}{ll}
0.028 & 0.006 \\
0.006 & 0.003
\end{array}\right], \\
& \boldsymbol{P}_{3}=\left[\begin{array}{ll}
0.0009 & 0.0007 \\
0.0007 & 0.0018
\end{array}\right], \boldsymbol{P}_{4}=\left[\begin{array}{ll}
0.00004 & 0.00004 \\
0.00004 & 0.0042
\end{array}\right]
\end{aligned}
$$

and the corresponding control gain matrices are obtained as $\boldsymbol{K}_{1}=\left[\begin{array}{ll}-27.07 & -1.45\end{array}\right], \quad \boldsymbol{K}_{2}=\left[\begin{array}{ll}-9.22 & -2.01\end{array}\right]$, $\boldsymbol{K}_{3}=\left[\begin{array}{ll}-2.77 & -2.05\end{array}\right]$, and $\boldsymbol{K}_{4}=\left[\begin{array}{ll}-2.16 & -1.92\end{array}\right]$ respectively. The size of the packet is assumed to be 500 bytes.

The numerical experiments are conducted on a network simulator-NS2 [14]. The simulation results with the chosen parameters show satisfactory performance of the queuing response in the presence of random delays, see Figure 4. It is found that larger TCP flows cause higher link utilizations, but with larger queuing delays.

The results for the case of $N_{1}=100, N_{2}=50$, $N_{3}=20, N_{4}=5$ are displayed in Figure 5. As it can be seen from Figure 5(a), large queue oscillations may cause considerable variations in the RTT of packets for the corresponding sub-network. However, with the proposed controllers, the network still remains to be stable when it holds a large number of data flows.

\section{Conclusions}

This paper has modeled and analyzed stability of largescale wired networks under control where the AQM strategy uses RED to fulfill the queue management. The problem of feedback control has been solved for the LWNS with delayed perturbations in the interconnections and a new condition ensuring the overall loop stability is 


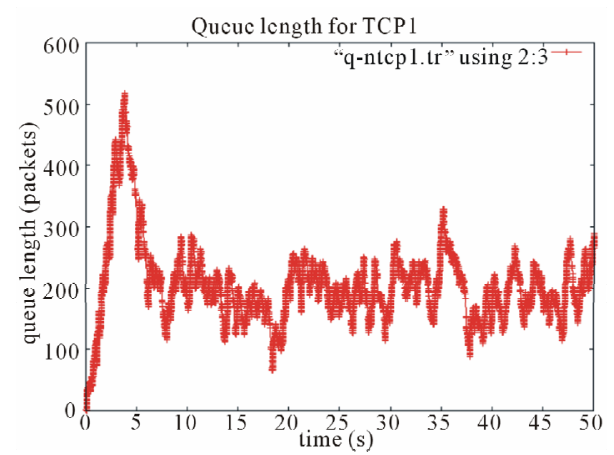

(a)

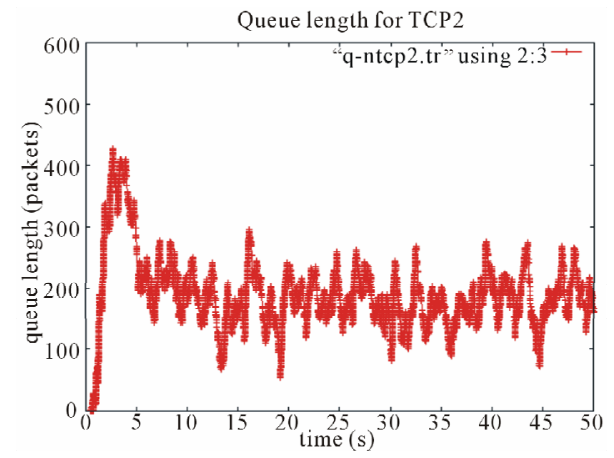

(b)

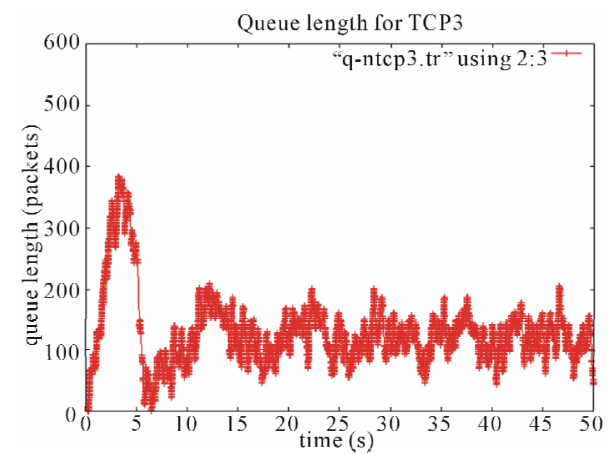

(c)

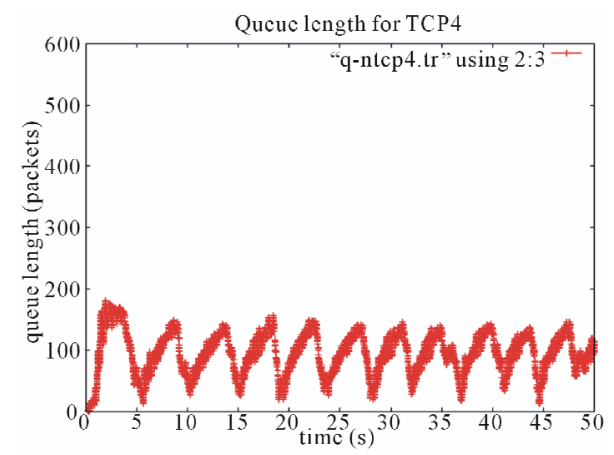

(d)

Figure 4. Transient response of queueing with $N_{1}=60$, $N_{2}=50, N_{3}=20$ and $N_{4}=5$. (a) Queue size for TCP1; (b) Queue size for TCP2; (c) Queue size for TCP3; (d) Queue size for TCP4.

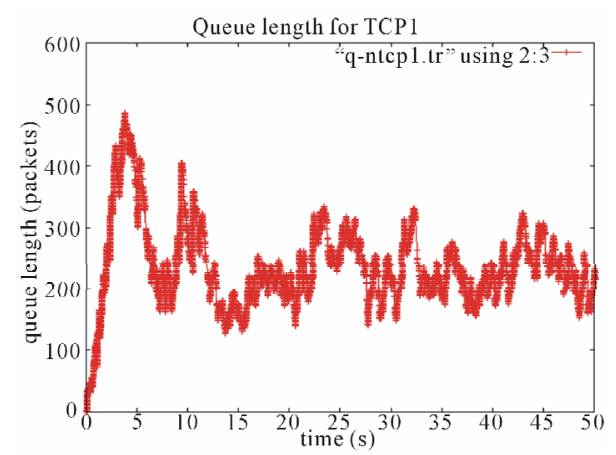

(a)

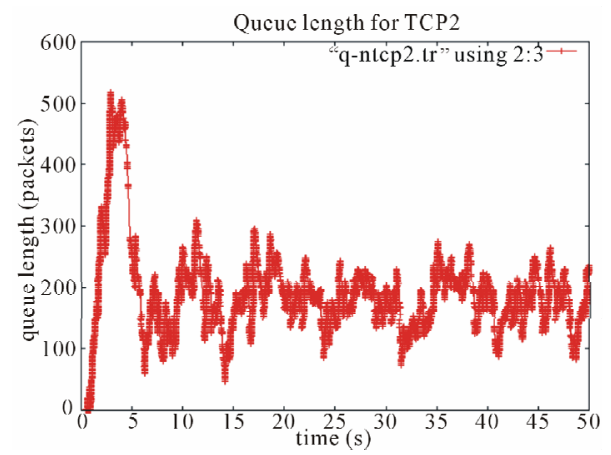

(b)

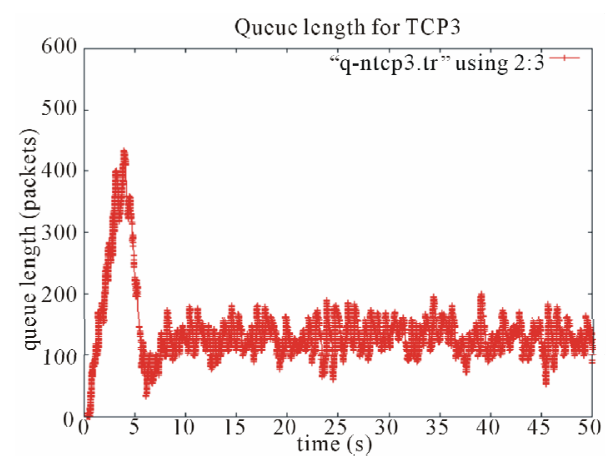

(c)

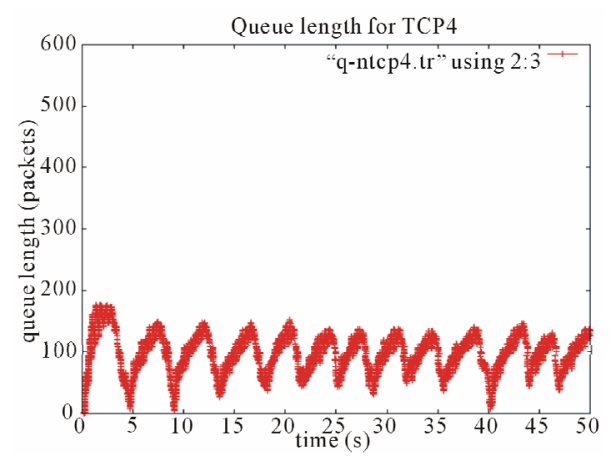

(d)

Figure 5. Transient response of queueing with $N_{1}=100$, $N_{2}=50, N_{3}=20$ and $N_{4}=5$. (a) Queue size for TCP1, (b) Queue size for TCP2; (c) Queue size for TCP3; (d) Queue size for TCP4. 
presented. The simulation study conducted on the NS2 has been verified successfully.

\section{Acknowledgements}

This research was sponsored by National Science Council, Taiwan under the grant NSC No. 95-2221-E-005-017.

\section{References}

[1] C. V. Hollot, V. Misra, D. Towsley and W. B. Gong, "Analysis and Design of Controllers for AQM Routers Supporting TCP Flows," IEEE Transactions on Automatic Control, Vol. 47, No. 6, 2002, pp. 945-959. doi:10.1109/TAC.2002.1008360

[2] V. Misra, W. B. Gong and D. Towsley, "Fluid-Based Analysis of a Network of AQM Routers Supporting TCP Flows with an Application to RED," Proceedings of ACM Special Interest Group on Data Communication, Stockholm, 28 August-2 September 2000, pp. 151-160.

[3] P. F. Quet and H. Ozbay, "On the Design of AQM Supporting TCP Flows Using Robust Control Theory," IEEE Transactions on Automatic Control, Vol. 49, No. 6, 2004, pp. 1031-1036. doi:10.1109/TAC.2004.829643

[4] B. A. Chiera and L. B. White, "A Subspace Predictive Controller for End-to-End TCP Congestion Control," Proceedings of Australian Communications Theory Workshop, Brisbane, 2-4 February 2005, pp. 42-48. doi:10.1109/AUSCTW.2005.1624224

[5] S. Tarbouriech, C. T. Abdallah and J. Chiasson, "Advances in Communication Control Networks," Springer, Berlin, 2005.

[6] K. Zhang and C. P. Fu, "Dynamics Analysis of TCP Veno with RED," Computer Communications, Vol. 30, No. 18, 2007, pp. 3778-3786. doi:10.1016/j.comcom.2007.09.004
[7] F. Zheng and J. Nelson, "An $H_{\infty}$ Approach to Congestion Control Design for AQM Routers Supporting TCP Flows in Wireless Access Networks," Computer Networks, Vol. 51, No. 6, 2007, pp. 1684-1704. doi:10.1016/j.comnet.2006.09.003

[8] Y. L. Wang and G. H. Yang, "State Feedback Control Synthesis for Networked Control Systems with Packet Dropout," Asia Journal of Control, Vol. 11, No. 6, 2009 , pp. 49-58. doi:10.1002/asjc.79

[9] H. B. Li, Z. Q. Sun, H. P. Liu and M. Y. Chow, "Predictive Observer-Based Control for Networked Control Systems with Network Induced Delay and Packet Dropout," Asia Journal of Control, Vol. 10, No. 6, 2008, pp. 638650. doi: $10.1002 /$ asjc. 65

[10] L. Shi, M. Epstein and R. M. Murray, "Control Over a Packet Dropping Network with Norm Bounded Uncertainties," Asia Journal of Control, Vol. 20, No. 1, 2008, pp. 14-23. doi:10.1002/asjc. 2

[11] H. Wu, "Decentralized Adaptive Robust Control for a Class of Large-Scale Systems Including Delayed State Perturbations in the Interconnections", IEEE Transactions on Automatic Control, Vol. 47, No. 10, 2002, pp. 1745-1751.

[12] F. H. Hsiao, J. D. Hwang, C. W. Chen and Z. R. Tsai, "Robust Stabilization of Nonlinear Multiple Time-Delay Large-Scale Systems via Decentralized Fuzzy Control," IEEE Transactions on Fuzzy Systems, Vol. 13, No. 1, 2005, pp. 152-163. doi:10.1109/TFUZZ.2004.836067

[13] H. Zhang, C. Li and X. Liao, "Stability Analysis and $H_{\infty}$ Controller Design of Fuzzy Large-Scale Systems Based on Piecewise Lyapunov Functions," IEEE Transactions on Systems, Man, and Cybernetics, Part B, Vol. 36, No. 3, 2006, pp. 685-698.

[14] DARPA, “The Network Simulator-NS-2,” 1995. http://www.isi.edu/nsnam/ 